\title{
Closed-loop electrical neurostimulation: Challenges and opportunities
}

\author{
Iñaki Iturrate*, Michael Pereira*, José del R. Millán
}

\begin{abstract}
Non-invasive and invasive electrical neurostimulation are promising tools to better understand brain function and ultimately treat its malfunction. In current open-loop approaches, a clinician chooses a fixed set of stimulation parameters, informed by observed therapeutic benefits and previous empirical evidence. However, this procedure leads to a large intra- and inter-subject variability often introducing side-effects and low effect sizes. Closed-loop electrical neurostimulation (CLENS) approaches strive to alleviate these limitations by tailoring the stimulation parameters to an ongoing electrophysiological biomarker. Here, we review the current status of closed-loop, supraspinal electrical stimulation in humans, presenting our vision of potential control frameworks, and support the idea of creating synergies with the field of brain-machine interfacing. Finally, we pinpoint two pivotal challenges that, in our view, need to be overcome for this technology to become a reality: dealing with the electrical stimulation artifacts, and dissociating the pathological from physiological information within the targeted biomarker.
\end{abstract}

\section{Acronyms}

Closed-loop electrical neurostimulation; deep brain stimulation; transcranial alternating current stimulation; transcranial direct current stimulation; electroencephalography; local field potentials.

\section{Acronyms}

$\begin{array}{ll}\text { CLENS Closed-loop electrical neurostimulation } \\ \text { DBS } & \text { Deep brain stimulation } \\ \text { ECOG } & \text { electrocorticography } \\ \text { EEG } & \text { electroencephalography } \\ \text { LDA } & \text { linear discriminant analysis } \\ \text { LFP } & \text { local field potentials } \\ \text { M1 } & \text { primary motor cortex } \\ \text { MEG } & \text { magnetoencephalography } \\ \text { PD } & \text { Parkinson's disease } \\ \text { STN } & \text { subthalamic nucleus } \\ \text { tACS } & \text { transcranial alternating current stimulation } \\ \text { tDCS } & \text { transcranial direct current stimulation } \\ \text { TMS } & \text { transcranial magnetic stimulation }\end{array}$




\section{Introduction}

Electrical neurostimulation is a promising tool to better understand brain function and ultimately treat its malfunction. Deep brain stimulation (DBS) in subcortical structures, such as the subthalamic nucleus (STN), the global pallidus or the thalamus, is now a wellestablished technique to treat refractory Parkinson's disease (PD), essential tremor, dystonia and obsessive-compulsive disorders [Budman2017]. Moreover, its use in other psychiatric diseases such as depression or addiction is being evaluated [Nuttin2014]. On the other hand, transcranial direct current stimulation (tDCS) has shown promising albeit limited effects on many neurological diseases such as stroke, aphasia, Alzheimer's disease or schizophrenia non-invasively [Lefaucheur2017].

While the field of neurostimulation has drastically advanced and attracted a growing interest over the last years, its limitations have also become more evident. First, little is known about the physiological effects of brain stimulation on the neuronal activity in the long term. As a result, stimulation parameters such as intensity, frequency, pulse shape and phase are chosen based solely on previous empirical evidence, and on the short-term adequacy of the stimulation in terms of the patient's clinical improvement [Sun2014]. Secondly, despite neural activity being highly non-stationary at the subsecond level, these stimulation parameters remain fixed throughout their clinical life, in an approach usually termed as openloop neurostimulation. Altogether, it is now widely believed that these issues are key limiting factors which lead to side-effects [Meidahl2017], a large intra- and inter-subject variability, a suboptimal efficacy of the stimulation treatment, and could partially explain some seemingly contradictory (or with a limited effect size) results in the literature [Thut2017].

In an ideal scenario, such limitations may be solved by the application of closed-loop electrical neurostimulation (CLENS). In CLENS, a pathological biomarker -an electrophysiological or biological correlate of the neurological condition to be treated- is constantly monitored, while the stimulation parameters are tailored by its fluctuations with the aim of regulating it [Hebb2014, Bergmann2016, Karabanov2016, Zrenner2016, Thut2017] (see Figure 1). Preliminary results with invasive stimulation suggest the potential benefits of such closed-loop technology [Little2013, Little2016], and current opinions are strongly in favor of this approach [Thut2017].

Here, we review the current status of closed-loop invasive and non-invasive neurostimulation in humans, with a purposeful focus on supraspinal electrical stimulation. In this opinion paper, we present our vision of potential control frameworks during brain-stimulation interactions in such closed-loop scenario, and support the idea of creating synergies with the field of brain-machine interfacing. Finally, we pinpoint two pivotal challenges that need to be overcome for this technology to become a reality: dealing with stimulation artifacts and understanding the neurophysiological mechanisms of stimulation, in particular the distinction between pathological and physiological information within the targeted biomarker.

\section{State of the art}

While invasive and non-invasive open-loop stimulation works are numerous, CLENS applications are still scarce, as they go hand-to-hand with advances in the understanding of 
the physiological effects of stimulation and the emergence of potential biomarkers for the targeted condition. Still, some works have already presented promising results, mostly using deep brain stimulation.

The first CLENS systems were designed to treat epilepsy, where the stimulation was delivered only when seizures were detected using an algorithm based on continuous ECoG recordings [Anderson2008]. Such a principle is now in clinical use by epileptic patients with favorable outcomes [Bergey2015, Geller2017]. Closed-loop DBS was also demonstrated on a primate model of PD [Rosin2011] and two years later in humans, where DBS stimulation in the STN was only switched on when beta oscillatory power was above a certain threshold [Little2013]. This led not only to a significant improvement of motor symptoms, but also to a $50 \%$ reduction in stimulation time, suggesting that CLENS could substantially save battery life. On a follow-up study from the same group, it has also been proved that such approach reduces side effects associated with DBS [Little2016]. Since then, these results have been replicated multiple times [e.g. Rosa2015, Arlotti2018]. New biomarkers are also being considered, such as gamma oscillations in the motor cortex $(\sim 60 \mathrm{~Hz})$ which correlates with dyskinesia, perhaps due to medication or DBS [Swann2016], and have been used as an indicator that stimulation intensity should be reduced [Swann2018]. Closed-loop DBS stimulation was also attempted in essential and dystonic tremor patients, using hand kinematics as a biomarker [Cagnan2016]. By locking high-frequency stimulation in the ventrolateral thalamus to the phase of the tremor measured with an accelerator, Cagnan and colleagues were able to achieve the same level of tremor reductions as state-of-the-art open-loop DBS but with only $42 \%$ of stimulation.

Apart from DBS for movement disorders, there are few reports of CLENS in humans. One study applied intracortical stimulation in the supplementary motor cortex when premovement gamma exceeded a threshold and observed a slowing of motor behavior [Moore2018]. Promising new works suggest that CLENS could be applied to cognitive diseases too. One recent study relied on high gamma activity, a biomarker of memory recall, to trigger medial temporal lobe stimulation in epilepsy patients undergoing intracranial monitoring [Ezzyat2018]. As this exemplary work illustrates, identification of reliable markers of cognitive disorders will lead to new avenues for CLENS. As new applications of open-loop DBS are being investigated, such as ventral striatum stimulation for obsessive compulsive disorder [Greenberg2010], depression [Kubu2017] or Alzheimer's disease [Scharre2018], and nucleus accumbens stimulation for traumatic brain injury [Rezai2016], the future will certainly see the extrapolation of these open-loop approaches to closed-loop scenarios [e.g. Senova2018, Bina2018].

Non-invasive stimulation has been mainly relying on direct currents applied on the scalp (tDCS). Nonetheless, many clinical trials have shown exciting yet limited clinical effects [Lefaucheur2017]. Recently, however, new alternating current strategies are being considered, the most notorious being transcranial alternating current stimulation (tACS), for which sinusoidal currents are applied to the scalp to selectively target a brain oscillation process [e.g. Feurra2011] or to modify cortical excitability [e.g. Moliadze2010]. Very few attempts to close the loop between stimulation parameters and ongoing brain oscillations have been reported. Applying tACS after sleep spindles were detected with EEG improved motor memory consolidation [Lustenberger2016]. Another recent closed-loop study also showed improvements in memory generalization when tACS during sleep was triggered by 
endogenous slow oscillations and matching their frequency and phase [Ketz2018]. Interestingly, $5 \mathrm{~Hz}$ tACS stimulation led to a $42 \%$ average reduction in tremor when the stimulation was set to be in-phase with the tremor [Brittain2013]. Albeit without closing the loop, other studies have shown the importance of phase timing when stimulating. Using two tACS stimulators either in-phase or out-of-phase, in-phase stimulation led to improved behavior [Polania2012]. Cortical excitability was also increased when pairing tACS bursts with peripheral afferent stimulation during in-phase stimulation [McNickle2015].

\section{Biomarker extraction and control strategies}

In a CLENS scenario, a biomarker is tracked in real-time and the stimulation parameters are tailored by this biomarker. A biomarker can be understood as an electrophysiological (invasive via electrocorticography or ECoG, local field potentials or LFPs, multispiking activity; or nor invasive, via electroencephalography or EEG) or biological correlate of the neurological condition to be treated, either as an epiphenomenon, or fashioning a certain physiological behavior. Usually, biomarkers extraction relies on the oscillatory nature of these signals. Alternatively, this biomarker can be extracted from indirect, behavioral methods, e.g. an accelerometer to measure tremor [Cagnan2016]. An important consideration is how to extract this biomarker reliably and in a subject-specific manner, a task where data-driven approaches may be superior (see Box 1).

Once the biomarker is extracted, it has to be mapped onto the stimulation parameter space. In general, the stimulation parameters that can be controlled are five: location, pulse shape, intensity (including on/off triggering), frequency, and phase. We distinguish between discrete and continuous mapping. In a discrete mapping, a certain stimulation parameter can only have a discrete set of values (e.g. on/off stimulation) based on the value of the biomarker (e.g. above or below a predefined threshold [Little2013, Swann2018]). Alternatively, the biomarker can be mapped continuously onto the range of values of one (eventually several) stimulation parameter(s), with the assumption that the tracked biomarker quantitatively encodes the symptoms. In PD patients, previous works have shown this to be the case [Rosa2015, Neumann2016, Martin2018]. Depending on the nature of the biomarker and the target stimulation parameter to be modulated, we have identified four control strategies (Figure 2).

\section{III.1) Biomarker amplitude - stimulation amplitude}

In this control strategy (Figure 2.1), the stimulation intensity is controlled by the ongoing amplitude/power of the biomarker. In this group of approaches, we include those that trigger on/off the stimulation. Little et al. triggered $130 \mathrm{~Hz}$ STN-DBS stimulation based on the oscillatory power of beta frequency in Parkinsonian patients [Little2013]; while Rosa et al. mapped the stimulation intensity continuously based on the continuous beta values [Rosa2015]. Alternatively, gamma power of ECoG recordings in the primary motor cortex (M1) can also be used to drive the STN-DBS for PD [Swann2018].

Previous works have shown the existence of oscillatory power biomarkers for other conditions, making them potential applications for this control strategy. Motor conditions such as dystonia are associated with beta increases over the STN [Wang2016], while tics associated with Tourette's syndrome are characterized by an increase in low frequencies 
over thalamus LFPs [Shute2016]. In spinal-cord injured patients, sensorimotor cortical mu rhythms inversely correlate with the severity of their symptoms [LopezLarraz2015], a physiological biomarker linked as well to corticospinal excitability [Thies2018]. Similarly, a range of cognitive disorders have been shown to have distinct pathological biomarkers. For instance, schizophrenia is characterized by pathological gamma synchronization [Uhlhaas2006], while Alzheimer leads to cortical delta and theta power decreases [Uhlhaas2006].

\section{III.2) Biomarker phase - stimulation amplitude}

In a second control strategy (Figure 2.2), certain phases of a biomarker tune or trigger the stimulation intensity. Such an approach has already been evaluated within closed-loop transcranial magnetic stimulation (TMS), where TMS pulses applied at certain oscillatory alpha phases led to different motor evoked potentials amplitudes [Zrenner2018]. Similarly, electrical stimulation intensity could be tuned based on the phase of the tracked biomarker. This approach has shown its efficacy for treating pathological tremor, using an accelerometer as a biomarker and a short-burst stimulation depending on the phase of the acceleration [Cagnan2016].

A more complex situation could involve tracking the activity of two brain regions simultaneously. In this approach, inter-regional interactions measured by the phase synchrony between them would drive the stimulation intensity. It is already well known that certain conditions lead to pathological inter-regional interactions. Such is the case of stroke and its associated pathological inter-hemispheric M1 interactions [Hummel2006], which have been seen to be correlated with clinical scores as measured by cortical coherence [Pichiorri2018]. In this case, a CLENS system could track interhemispheric coherence and stimulate one or both brain regions accordingly [Hummel2006].

\section{III.3) Biomarker phase - stimulation phase}

This control strategy aims at driving the phase of a biomarker by altering the stimulation phase (Figure 2.3). One such example was provided by Brittain et al., where the tracked pathological tremor phase was used to tune non-invasive tACS anti-phase to the biomarker [Brittain2013]. Another case is when one aims at cancelling oscillatory power by stimulating in anti-phase. Following the example of pathological increase in beta power during PD, its phase could be tracked and used to stimulate in anti-phase. Yet another potential scenario is provided by the work of Tran et al., who showed that pre-stimuli alpha phase correlates with memory decline [Tran2016]. A CLENS approach based on this biomarker could, for instance, stimulate at specific phases to alter the alpha-phase biomarker.

This approach could also be beneficial for inter-regional approaches. Fries suggested that neuronal communication is subserved by neural synchronization within different frequency ranges [Fries2015]. As such, pathologies with network-based biomarkers may benefit from this control strategy, where the oscillatory phase of one brain region tailors the stimulation phase over a second brain region. 


\section{III.4) Other biomarkers and stimulation parameters}

While most of the research on biomarkers has been done using oscillatory features (amplitude and phase), other biomarkers may prove to be superior in certain conditions. For instance, one such biomarker could be evoked activity such as event-related potentials (Figure 2.4), which can be decoded at the single-trial level using pattern recognition [e.g. Iturrate2015]. Along these lines, a CLENS system was developed based on the decoding of evoked activity in the frequency domain [Ezzyat2018]. Other examples include the use of phase-amplitude coupling, which could be a promising biomarker for PD [deHemptinne2015]; or the total power of the broadband spectrum, which has been suggested to be linked to schizophrenia [Peterson2017]. Finally, as single neuron activity is being recorded in human patients during DBS surgery [e.g. Milosevic2018] and epilepsy monitoring [e.g. Wang2018], it is easy to foresee future biomarkers based on firing rates of single neurons.

\section{Challenges}

Although the field seems to agree that CLENS is a promising future for brain stimulation, it is still facing a number of challenges. Here, we focus on two challenges that seem paramount to us; how to deal with the stimulation artifact, and how to dissociate pathological from physiological brain activity when computing the biomarkers.

\section{IV.1) Dealing with the stimulation artifacts}

One major limitation of CLENS is the presence of a massive stimulation artifact on electrophysiological (or magnetophysiological) recordings. These artifacts can have different properties depending on the tissues stimulated, the stimulation pattern and the type of recordings and their location with respect to the stimulation site.

In some situations, biomarkers can be recovered without removing the artifact. Through appropriate filtering, beta oscillations can be recorded while stimulating with DBS at much higher frequencies [Tinkhauser2017], yet higher frequencies with potential information are unavailable. Another solution is to record from other parts of the brain [Swann2018] or even from biomarkers such as kinematics [Cagnan2016], although these solutions drastically limit the types of biomarkers that can be used. For non-invasive tACS, the brain oscillation of interest is often within the frequency of stimulation and thus extremely difficult to recover.

One first step in order to reduce artifacts is to optimize the system design. Research on animal models have made technical advances to reduce artifacts before the signal is digitized [Zhou2018]. For example, indirect artifacts due to the capacitance of the electrodes and tissues can be reduced by ensuring that the positive and negative pulses have equal current amplitude [Johnson2017] or by careful selection of stimulation and recording parameters [Stanslaski2012]. Others have modulated the stimulation signal at a higher frequency [Witkowski2016] to recover the underlying brain signal using magnetoencephalography (MEG).

Various methods have been employed to remove the artifact after digitization. MEG signals during tACS have been recovered using beamforming [Neuling2015, Kasten2018], but see 
[Mäkelä2017]. Others have decomposed the contaminated electrophysiological signal into subspaces and rejected those subspaces that represented the stimulation artifact [Hofmanis2013, Helfrich2014]. Alternatively, an artifact template can be computed and subtracted to recover the underlying brain signal. One way of computing the artifact template is by averaging a sufficient amount of time-locked stimulation artifacts patterns [Sun2016]. Such systems, however, have difficulties adapting to fast changes of the artifact which could be typical in CLENS. Moreover, for tACS, an additional offline processing step had to be performed to completely remove the residual artifact [Helfrich2014], which impedes its use in real-time applications. More appropriately for real-time applications, one can compute the stimulation artifact template in real-time from a readout of the stimulator before the current reaches the brain [Trebaul2016].

Two issues can affect these template subtraction methods. Firstly, they can only work under the assumption that the stimulation artifact linearly sums up to the underlying brain activity, which in turn implies that the signal stays within the linear range of the amplifier [Rolston2009]. It has indeed been suggested that electrical stimulation interacts with electrophysiological signals in a non-linear fashion, with other physiological processes such as heartbeat or respiration being potential factors [Noury2016, Neuling2017], although models are starting to be proposed [Noury2017]. Secondly, when the frequency of stimulation lies within the frequency of the biomarker, brain oscillations can entrain (i.e. phase-lock) to the stimulation and lead to erroneous recovery (Box 2).

Although recovering from stimulation artifacts seem feasible for most electrical neurostimulation modalities, this is not obvious for others. This is particularly the case for transcranial random noise stimulation, a variant of tACS, where alternating currents are applied in a very wide range of frequencies (typically between 0 and $1000 \mathrm{~Hz}$ ).

\section{IV.2) Biomarker: Pathology or physiology?}

Understanding the actual impact that electrical stimulation has at the electrophysiological and behavioral level remains one of the most important challenges in the field. Although the topic has been covered extensively elsewhere [Fertonani2017, Herrmann2017, Harmsen2018] for open-loop stimulation, there is scarce knowledge on the neurophysiological implications of CLENS (see [Tinkhauser2017] for an exception). One crucial challenge is the fact that the biomarker controlling the stimulation parameters will most often represent an overlap of both pathological activity and healthy physiological processes, thus leading to an incorrect stimulation triggering and tampering with physiological behavior.

Usually, biomarkers are just considered as a proxy to an ongoing pathology. Still, these biomarkers are also associated with physiological behavior. Such is, for instance, the case for pathological beta power during PD. Although CLENS for PD has shown its efficacy using clinical measures [Little2013, Rosa2015], a recent work has relativized the benefits of betadriven CLENS, suggesting that its advantages over open-loop stimulation can be compromised under certain conditions [Johnson2016]. During the experiment, a non-human primate had to perform a goal-oriented reaching task, which is known to desynchronize beta activity. This reduction in beta led to switching off the stimulation, and in turn to a worsening of the behavioral outcomes during reaching compared to continuous stimulation. Although 
no work has quantified its impact for human PD patients, it is reasonable to believe that this effect will stand true as volitional movements lead to modulations in these frequencies, e.g. during gait [Hell2018] and upper-limb movements [Kühn2004].

In general, one can expect this to be an important consideration for any CLENS application using brain-decoded biomarkers. A promising solution is to use data-driven, classification approaches (cf. Box 1) with the aim of finding a more robust biomarker (or a secondary biomarker) that helps distinguishing the context in which the subject currently is. An example is provided by Shute et al., where they used pattern recognition to distinguish between pathological tics and voluntary movements in Tourette's syndrome during concurrent M1STN LFP recordings: while both shared some electrophysiological characteristics (beta desynchronization over M1), pathological tics were also encoded as low-frequency power increases over the thalamus [Shute2016].

\section{Conclusion}

A growing number of studies are using closed-loop approaches to improve the limitations of open-loop electrical neurostimulation. Yet, while most reviewed studies use CLENS to shorten stimulation time and thus potentially reduce side-effects, few studies have shown behavioral improvements over open-loop stimulation. Here, we have reviewed evidence suggesting that new control strategies could enhance effect sizes but that two major challenges still need to be addressed; firstly, the stimulation artifact needs to be removed from the physiological recordings in order to access a broader range of possible biomarkers which can then be used to develop more complex control strategies. Secondly, in order to continuously adapt the stimulation parameters, there is a need to dissociate physiological activity from the actual pathological biomarker that drives the stimulation. To overcome current limitations, the field will certainly benefit from synergies with the brain-machine interface field.

There are certainly other challenges for CLENS to bring to fruition its full potential. One of them is at the heart of the fundamental hypothesis of CLENS, namely that there is a causal relationship between the chosen biomarker and the pathology to be treated, and that regulating this biomarker will improve patient's clinical conditions. Finding the correct biomarker will require better understanding of how the brain works in the healthy and pathological conditions. While neuroscience, neurology and related clinical researchers may lead this enterprise, contributions from biomedical engineering will still play crucial roles in building computational models to help discover the target biomarker, or even combinations of biomarkers. Such models will extend current open-loop approaches [Bikson2012, MolaeeArdekani2013].

Data-driven, especially if coupled to brain-machine interfaces, CLENS approaches promise to become a fundamental framework, not only to develop novel symptomatic and rehabilitative therapies, but also to answer basic physiological questions. Furthermore, we envision CLENS to replace the more empirical open-loop approaches, and become a crucial research tool to better understand the brain's physiology. This will be even more the case once CLENS will be deployed for long-term use, where detection of the target biomarker and choice of parameters of the control strategy must be continuously adapted to reflect evolution of patients' clinical conditions. 


\section{Box 1 - Biomarker decoding: A brain-machine interface perspective}

An important limitation of current CLENS approaches is the empirical nature of the parameters chosen, which can be softened by using subject-specific, data-driven models. In this regard, the field of brain-machine interfaces (BMIs) provides unique knowledge for the development of successful closed-loop stimulation strategies. In a BMI, electrophysiological signals are tracked in real time and they serve as control commands for a neural prosthesis, which in turns provides feedback to the user about their performance (see [Millán2010] for a comprehensive review). Similarly, CLENS can be seen as a synergy between a BMI and typical open-loop stimulation, where usual BMI feedback is replaced by electrical stimulation. In sum, two personalized components can be distinguished: feature extraction and selection that will encode the biomarker; and classification of these features for its mapping to the stimulation parameters.

First, features are extracted from the raw electrophysiological recordings either within the temporal or spectral domain. As this feature extraction usually leads to a large number of features, measures of features discriminability are needed to derive a subset of features that maximize the performance for each subject, based on a personalized analysis. For this process to be effective, a supervised training session is required, where electrophysiological recordings are labeled in a controlled situation (e.g. symptoms vs no symptoms conditions). This method will lead to the set of features that will encode the biomarker used during CLENS. In the most common case of two labeled conditions, the classical discriminability measure is the Fisher score, defined as follows:

$$
f s(f)=\frac{\mu_{1}(f)-\mu_{2}(f)}{\sigma_{1}(f)+\sigma_{2}(f)}
$$

where $\mu_{i}(f), \sigma_{i}(f)$ represent the mean and standard deviation of condition $i$ of one feature $f$, e.g. the oscillatory power at one particular frequency.

Once features are extracted, they are fed into a classification or regression algorithm. The most common method due to its simplicity and flexibility is the linear discriminant (LDA), where the output is simply a linear combination of the features:

$$
y=w x+b
$$

where $x$ is the vector of features, and $w$ and $b$ the LDA coefficients. The sign of $y$ encodes the predicted condition for the current feature vector, which can be directly linked to a discrete mapping of a stimulation parameter (see section III of the manuscript for details). Alternatively, it can be used for a continuous mapping by simply applying a sigmoid function to the output, which fits the output within a constrained range:

$$
\sigma(y)=\frac{1}{1+e^{-b y}}
$$

where $b$ controls the slope of the sigmoid. 


\section{Box 2 - Artifact removal with entrainment}

Prospective artifact removal methods should take in consideration the entrainment of electrophysiological signals. There is mounting evidence that brain oscillations entrain to periodic currents such as tACS [Amengual2017] or DBS [Swann2016] in a similar way as they entrain to rhythmic stimuli [Lakatos2008] or rhythmic TMS pulses [Thut2011].

We exemplify the problem that can arise due to entrainment with concurrent tACS-EEG recordings in the alpha band $(\sim 10 \mathrm{~Hz})$. To remove the artifact, a template is constructed using a copy of the output of the stimulator. This template is then scaled and corrected for phase shifts before being subtracted to the contaminated signal. The scaling and phase correction parameters are computed during a calibration phase. If, due to entrainment, the EEG is phase-locked to the tACS stimulation, the in-phase EEG component will affect the scaling factor while other components will affect the phase correction factor. To prevent entrainment during calibration it can be useful to use a succession of stimulation bursts rather than continuous stimulation. The bursts should be short enough to ensure that there is no entrainment and that the stimulation signal is independent from the brain oscillations. They should also be numerous enough to ensure that the effect of differences in phase between the brain oscillations and the stimulation cancel out. Using such a calibration procedure, we were able to recover alpha oscillations while stimulating with HD-tACS at $1 \mathrm{~mA}(\mathbf{A})$.

Since CLENS main component is that of tracking in real-time a targeted biomarker, we argue that artifact removal evaluation methods should consider the use of single-trial decoders to test for the efficacy of the artifact cleaning procedure. During an event-related potential task [Iturrate2015], we calibrated the coefficients every minute and were able to obtain similar decoding performance during tACS at $1 \mathrm{~mA}$ than before; yet, after stimulation, and because of the entrainment effect, decoding performance improved (B). The use of single-trial pattern recognition as an evaluation tool after artifact removal, rather than a modeling perspective, provides us with a functional, application-driven indicator of the feasibility of CLENS for the targeted biomarker.

A

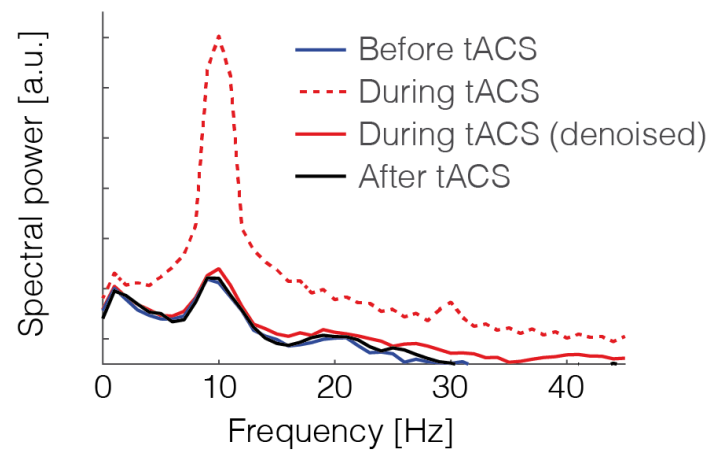

$\mathrm{B}$

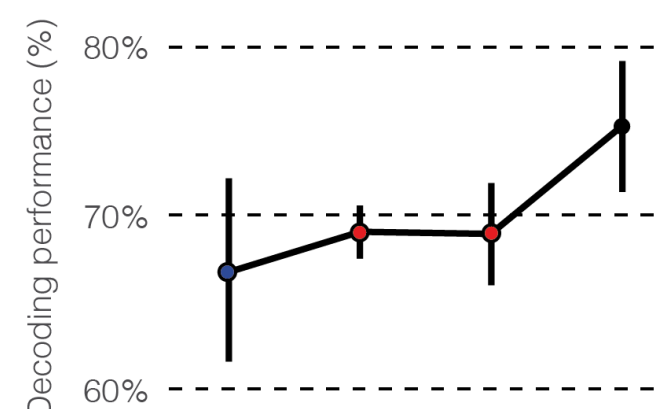

Before tACS During tACS After tACS 


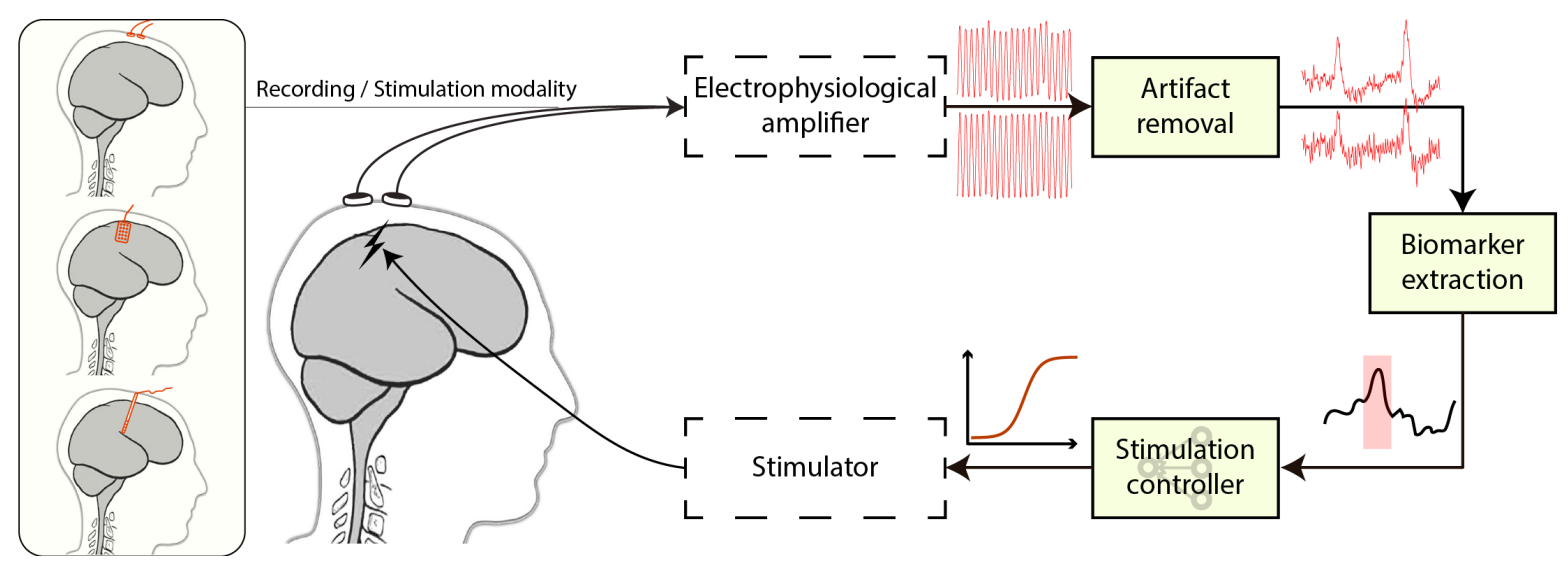

Figure 1. Closed-loop electrical neurostimulation (CLENS). Contrarily to open-loop stimulation where the stimulation is applied in a fixed manner, in CLENS the stimulation parameters are tailored based on the presence of extracted biomarkers. The recording and stimulation modalities can be a combination of three modalities: non-invasively via electroencephalography, or invasively via cortical electrocorticography or subcortical local field potentials with deep electrodes (see left box). For a CLENS to be achieved, we distinguish three main modules (depicted in green): biomarker extraction, stimulation controller, and the removal or rejection of artifacts generated by the electrical stimulator on the electrophysiological signal. 


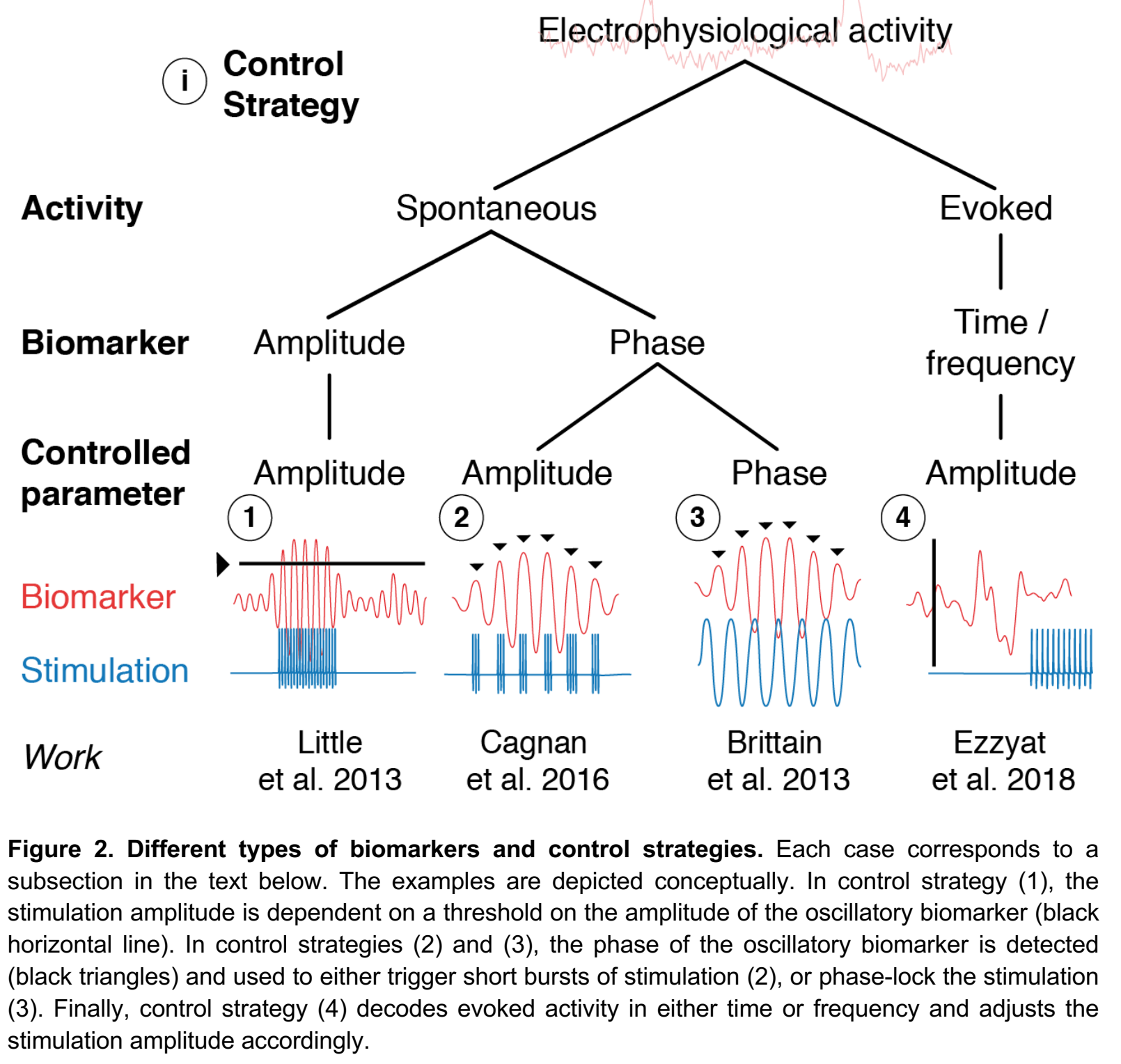




\section{Acknowledgements}

This work has been partially supported by the Swiss National Centres of Competence in Research (NCCR) Robotics (J.d.R.M.). I.I. acknowledges support from the 'EPFL Fellows' fellowship programme co-funded by Marie Curie, FP7 Grant agreement no. 291771.

\section{Conflict of interest}

The authors declare no conflict of interest. 


\section{References}

Amengual JL, Vernet M, Adam C, Valero-Cabré A (2017) Local entrainment of oscillatory activity induced by direct brain stimulation in humans. Sci Rep 7:e41908.

Anderson WS, Kossoff EH, Bergey GK, Jallo GI (2008) Implantation of a responsive neurostimulator device in patients with refractory epilepsy. Neurosurg Focus 25:E12.

Arlotti M, Marceglia S, Foffani G, Volkmann J, Lozano AM, Moro E, Cogiamanian F, Prenassi M, Bocci T, Cortese F, Rampini P, Barbieri S, Priori A (2018) Eight-hours adaptive deep brain stimulation in patients with Parkinson disease. Neurology 90: e971-e976.

Bergey GK et al. (2015) Long-term treatment with responsive brain stimulation in adults with refractory partial seizures. Neurology 84:810-817.

Bergmann T, Karabanov A, Hartwigsen G, Thielscher A, Siebner HR (2016) Combining non-invasive transcranial brain stimulation with neuroimaging and electrophysiology: current approaches and future perspectives. Neuroimage 140:4-19.

Beuter A, Lefaucheur JP, Modolo J (2014) Closed-loop cortical neuromodulation in Parkinson's disease: an alternative to deep brain stimulation? Clin Neurophysiol 125:874-885.

Bikson M, Rahman A, Datta A (2012) Computational models of transcranial direct current stimulation. Clin EEG Neurosci 43:176-183.

Bina RW, Langevin JP (2018) Closed loop deep brain stimulation for PTSD, addiction, and disorders of affective facial interpretation: review and discussion of potential biomarkers and stimulation paradigms. Front Neurosci 12:1-13.

Brittain JS, Probert-Smith P, Aziz TZ, Brown P (2013) Tremor suppression by rhythmic transcranial current stimulation. Curr Biol 23:436-440.

Budman E, Deeb W, Martinez-Ramirez D, Pilitsis JG, Peng-Chen Z, Okun MS, Ramirez-Zamora A (2018) Potential indications for deep brain stimulation in neurological disorders: an evolving field. Eur J Neurol 25:434-e30.

(••) Cagnan H, Pedrosa D, Little S, Pogosyan A, Cheeran B, Aziz T, Green A, Fitzgerald J, Foltynie T, Limousin P, Zrinzo L, Hariz M, Friston KJ, Denison T, Brown P (2016) Stimulating at the right time: phase-specific deep brain stimulation. Brain 140:132-145.

This paper demonstrates the efficacy of phase-specific DBS stimulation. By phase-locking stimulation of the ventromedial thalamus to the phase of patient's tremor, the authors achieved a reduction in tremor while reducing the total energy delivered by a factor of more than two compared to conventional DBS.

De Hemptinne C, Swann NC, Ostrem JL, Ryapolova-Webb ES, San Luciano M, Galifianakis NB, Starr PA (2015) Therapeutic deep brain stimulation reduces cortical phase-amplitude coupling in Parkinson's disease. Nat Neurosci 18:779-786.

(••) Ezzyat $Y$ et al. (2018) Closed-loop stimulation of temporal cortex rescues functional networks and improves memory. Nat Commun 9: e365.

This study used patients implanted for clinical monitoring of epilepsy to train a multivariate classifier to discriminate the encoding of words that were later recalled versus words that were later forgotten. Direct cortical stimulation of the medial temporal lobe was triggered when the output of this classifier on new stimuli was low, predicting low quality encoding of a word. This closed-loop procedure led to better recall of words.

Fertonani A, Miniussi C (2017) Transcranial electrical stimulation: what we know and do not know about mechanisms. Neurosci 23:109-123.

Feurra M, Bianco G, Santarnecchi E, Del Testa M, Rossi A, Rossi S (2011) Frequency-dependent tuning of the human motor system induced by transcranial oscillatory potentials. J Neurosci 31:12165-12170.

Fries P (2015) Rhythms for cognition: communication through coherence. Neuron 88:220-235.

(••) Geller EB, Skarpaas TL, Gross RE, Goodman RR, Barkley GL, Bazil CW, Berg MJ, Bergey GK, Cash SS, Cole AJ, Duckrow RB, Edwards JC, Eisenschenk S, Fessler J, Fountain NB, Goldman AM, Gwinn RP, Heck C, Herekar A, Hirsch LJ, Jobst BC, King-Stephens D, Labar DR, Leiphart JW, Marsh WR, Meador KJ, Mizrahi EM, Murro AM, Nair DR, Noe KH, Park YD, Rutecki PA, Salanova V, Sheth RD, Shields DC, Skidmore C, Smith MC, Spencer DC, Srinivasan S, Tatum W, Van Ness PC, Vossler DG, Wharen RE Jr, Worrell GA, Yoshor D, Zimmerman RS, Cicora K, Sun FT, Morrell MJ (2017) Brain-responsive neurostimulation in patients with medically intractable mesial temporal lobe epilepsy. Epilepsia. 58:994-1004.

Authors report how their implantable closed-loop stimulator, based on the detection of upcoming epileptic seizures from local field potentials, leads to a median seizure reduction of $70 \%$ after $6.1 \pm 2.2$ years in a cohort of 111 patients suffering from intractable unilateral or bilateral mesial temporal lobe epilepsy.

Greenberg BD et al. (2010) Deep brain stimulation of the ventral internal capsule/ventral striatum for obsessive-compulsive disorder: worldwide experience. Mol Psychiatry 15:64-79.

Harmsen IE, Rowland NC, Wennberg RA, Lozano AM (2018) Characterizing the effects of deep brain stimulation with magnetoencephalography: a review. Brain Stimul 11:481-491.

Hebb AO, Zhang JJ, Mahoor MH, Tsiokos C, Matlack C, Chizeck HJ, Pouratian N (2014) Creating the feedback loop: closedloop neurostimulation. Neurosurgery Clinics 25:187-204. 
Helfrich RF, Schneider TR, Rach S, Trautmann-Lengsfeld SA, Engel AK, Herrmann CS (2014) Entrainment of brain oscillations by transcranial alternating current stimulation. Curr Biol 24:333-339.

Hell F, Plate A, Mehrkens JH, Bötzel K (2018) Subthalamic oscillatory activity and connectivity during gait in Parkinson's disease. Neurolmage: Clinical 19:396-405.

Herrmann CS, Strüber D (2017) What can transcranial alternating current stimulation tell us about brain oscillations? Curr Behav Neurosci Reports 4:128-137.

Hofmanis J, Caspary O, Louis-Dorr V, Ranta R, Maillard L (2013) Denoising depth EEG signals during DBS using filtering and subspace decomposition. IEEE Trans Biomed Eng 60:2686-2695.

Hummel FC, Cohen LG (2006) Non-invasive brain stimulation: a new strategy to improve neurorehabilitation after stroke?. The Lancet Neurology 5:708-712.

Iturrate I, Chavarriaga R, Montesano L, Minguez J, Millán JdR (2015) Teaching brain-machine interfaces as an alternative paradigm to neuroprosthetics control. Sci Rep 5:13893.

(••) Johnson LA, Nebeck SD, Muralidharan A, Johnson MD, Baker KB, Vitek JL (2016) Closed-loop deep brain stimulation effects on Parkinsonian motor symptoms in a non-human primate - is beta enough? Brain Stimul 9:892896.

Using a primate model of PD, this study shows that contrarily to open-loop DBS, closed-loop DBS triggered by beta power fails to improve bradykinesia during movements, due to the decrease of beta preceding movement which is wrongly considered as a good biomarker state by the closed-loop system.

Johnson BC, Gambini S, Izyumin I, Moin A, Zhou A, Alexandrov G, Santacruz SR, Rabaey JM, Carmena JM, Muller R (2017) An implantable $700 \mu \mathrm{W} 64$-channel neuromodulation IC for simultaneous recording and stimulation with rapid artifact recovery. IEEE Symp VLSI Circuits, Dig Tech Pap C48-C49.

Karabanov A, Thielscher A, Siebner HR (2016) Transcranial brain stimulation: closing the loop between brain and stimulation. Current Opinion in Neurology, 29:397-404.

Kasten FH, Kasten FH, Maess B, Herrmann CS (2018) Facilitated event-related power-modulations during transcranial alternating current stimulation ( tACS ) revealed by concurrent tACS-MEG. eNeuro, 5:ENEURO.0069-18.2018.

Ketz N, Jones A, Bryant N, Clark VP, Pilly PK (2018) Closed-loop slow-wave tACS improves sleep dependent long-term memory generalization by modulating endogenous oscillations. J Neurosci 38:7314-7326.

Kubu CS, Brelje T, Butters MA, Deckersbach T, Malloy P, Moberg P, Tröster Al, Williamson E, Baltuch GH, Bhati MT, Carpenter LL, Dougherty DD, Howland RH, Rezai AR, Malone DA (2017) Cognitive outcome after ventral capsule/ventral striatum stimulation for treatment-resistant major depression. J Neurol Neurosurg Psychiatry 88:262-265.

Kühn AA, Williams D, Kupsch A, Limousin P, Hariz M, Schneider GH, Yarrow K, Brown P (2004) Event-related beta desynchronization in human subthalamic nucleus correlates with motor performance. Brain 127:735-746.

Lakatos P, Karmos G, Mehta AD, Ulbert I, Schroeder CE (2008) Entrainment of neuronal oscillations as a mechanism of attentional selection. Science 320:110-113.

Lefaucheur J-P et al. (2017) Evidence-based guidelines on the therapeutic use of transcranial direct current stimulation (tDCS). Clin Neurophysiol 128:56-92.

Little S, Pogosyan A, Neal S, Zavala B, Zrinzo L, Hariz M, Foltynie T, Limousin P, Ashkan K, Fitzgerald J, Green AL, Aziz TZ, Brown P (2013) Adaptive deep brain stimulation in advanced Parkinson Disease. Ann Neurol 74:449-457.

(••) Little S, Tripoliti E, Beudel M, Pogosyan A, Cagnan H, Herz D, Bestmann S, Aziz T, Cheeran B, Zrinzo L, Hariz M, Hyam J, Limousin P, Foltynie T, Brown P (2016) Adaptive deep brain stimulation for Parkinson's disease demonstrates reduced speech side effects compared to conventional stimulation in the acute setting. J Neurol Neurosurg Psychiatry $87: 1388-1389$.

This study was the first to demonstrate in human patients the feasibility of triggering DBS stimulation based on subthalamic beta power. The authors achieved similar clinical improvement while stimulating less time.

López-Larraz E, Montesano L, Gil-Agudo Á, Minguez J, Oliviero A (2015) Evolution of EEG motor rhythms after spinal cord injury: a longitudinal study. PloS One 10: e0131759.

(••) Lustenberger C, Boyle MR, Alagapan S, Mellin JM, Vaughn B V., Fröhlich F (2016) Feedback-controlled transcranial alternating current stimulation reveals a functional role of sleep spindles in motor memory consolidation. Curr Biol 26:2127-2136.

One of the few closed-loop studies using non-invasive stimulation. tACS stimulation was applied when sleep spindles were detected, thereby selectively increasing sigma activity and enhancing memory consolidation.

Martin S, Iturrate I, Chavarriaga R, Leeb R, Sobolewski A, Li AM, Zaldivar J, Peciu-Florianu I, Pralong E, Castro-Jimenez M, Benninger D, Vingerhoets F, Knight RT, Bloch J, Millán JdR (2018) Differential contributions of subthalamic beta rhythms and neural noise to Parkinson motor symptoms. bioRxiv 312819

Mäkelä, N., Sarvas, J., \& IImoniemi, R. J. (2017). A simple reason why beamformer may (not) remove the tACS-induced artifact in MEG. Brain Stimulation 10:e66-e67.

McNickle, E., \& Carson, R. G. (2015). Paired associative transcranial alternating current stimulation increases the excitability of corticospinal projections in humans. J Physiol 593:1649-1666. 
Meidahl AC, Tinkhauser G, Herz DM, Cagnan H, Debarros J, Brown P (2017) Adaptive deep brain stimulation for movement disorders: the long road to clinical therapy. Mov Disord 32:810-819.

Millán JdR, Rupp R, Müller-Putz GR, Murray-Smith R, Giugliemma C, Tangermann M, Vidaurre C, Cincotti F, Kübler A, Leeb R, Neuper C, Müller K-R, Mattia D (2010) Combining brain-computer interfaces and assistive technologies: state-of-the-art and challenges. Front Neurosci 4:1-15.

Milosevic L, Kalia SK, Hodaie M, Lozano AM, Popovic MR, Hutchison WD (2018) Physiological mechanisms of thalamic ventral intermediate nucleus stimulation for tremor suppression. Brain 141:2142-2155.

Molaee-Ardekani B, Márquez-Ruiz J, Merlet I, Leal-Campanario R, Gruart A, Sánchez-Campusano R, Birot G, Ruffini G, Delgado-García JM, Wendling F (2013) Effects of transcranial Direct Current Stimulation (tDCS) on cortical activity: a computational modeling study. Brain Stimulation 6:25-39.

Moliadze V, Antal A, Paulus W (2010) Boosting brain excitability by transcranial high frequency stimulation in the ripple range. J Physiol 588:4891-4904.

Moore BD, Aron AR, Tandon N (2018) Brain stimulation closed-loop intracranial stimulation alters movement timing in humans. Brain Stimul 11:886-895.

Neuling T, Ruhnau P, Fuscà M, Demarchi G, Herrmann CS, Weisz N (2015) Friends, not foes: magnetoencephalography as a tool to uncover brain dynamics during transcranial alternating current stimulation. Neuroimage 118:406-413.

Neuling T, Ruhnau P, Weisz N, Herrmann CS, Demarchi G (2017) Faith and oscillations recovered: on analyzing EEG/MEG signals during tACS. Neuroimage 147:960-963.

Neumann W-J, Degen K, Schneider G-H, Brücke C, Huebl J, Brown P, Kühn AA (2016) Subthalamic synchronized oscillatory activity correlates with motor impairment in patients with Parkinson's disease. Mov Disord 31:1748-1751.

Noury N, Hipp JF, Siegel M (2016) Physiological processes non-linearly affect electrophysiological recordings during transcranial electric stimulation. Neuroimage 140:99-109.

(•) Noury N, Siegel M (2017) Phase properties of transcranial electrical stimulation artifacts in electrophysiological recordings. Neuroimage 158:406-416.

A model of tACS artifact is proposed, characterizing phase properties of the tACS artifact and demonstrating frequencydependent non-linear interactions between non-invasive periodic stimulation and stimulation artifact.

Nuttin B et al. (2014) Consensus on guidelines for stereotactic neurosurgery for psychiatric disorders. J Neurol Neurosurg Psychiatry 85:1003-1008.

Peterson EJ, Rosen BQ, Campbell AM, Belger A, Bradley B (2017) 1/f neural noise is a better predictor of schizophrenia than neural oscillations. bioRxiv 113449.

Pichiorri F, Petti M, Caschera S, Astolfi L, Cincotti F, Mattia D (2018) An EEG index of sensorimotor interhemispheric coupling after unilateral stroke: clinical and neurophysiological study. Eur J Neurosci 47:158-163.

Polanía R, Nitsche MA, Korman C, Batsikadze G, Paulus W (2012) The importance of timing in segregated theta phasecoupling for cognitive performance. Curr Biol 22:1314-1318.

Rezai AR, Sederberg PB, Bogner J, Nielson DM, Zhang J, Mysiw WJ, Knopp M V., Corrigan JD (2016) Improved function after deep brain stimulation for chronic, severe traumatic brain injury. Neurosurgery 79:204-210.

Rolston JD (2009) A low-cost multielectrode system for data acquisition enabling real-time closed-loop processing with rapid recovery from stimulation artifacts. Front Neuroeng 2:1-17.

Rosa M, Arlotti M, Ardolino G, Cogiamanian F, Marceglia S, Di Fonzo A, Cortese F, Rampini PM, Priori A (2015) Adaptive deep brain stimulation in a freely moving parkinsonian patient. Mov Disord 30:1003-1005.

Rosin B, Slovik M, Mitelman R, Rivlin-Etzion M, Haber SN, Israel Z, Vaadia E, Bergman H (2011) Closed-loop deep brain stimulation is superior in ameliorating parkinsonism. Neuron 72:370-384.

Scharre DW, Weichart E, Nielson D, Zhang J, Agrawal P, Sederberg PB, Knopp M V., Rezai AR (2018) Deep brain stimulation of frontal lobe networks to treat Alzheimer's disease. J Alzheimer's Dis 62:621-633.

Senova S, Chaillet A, Lozano AM (2018) Fornical closed-loop stimulation for Alzheimer's disease. Trends Neurosci 41:418428.

(•) Shute JB, Okun MS, Opri E, Molina R, Rossi PJ, Martinez-Ramirez D, Foote KD, Gunduz A (2016) Thalamocortical network activity enables chronic tic detection in humans with Tourette syndrome. Neurolmage Clin 12:165-172. This offline study showed that while beta oscillatory activity in the cortex due to Tourette syndrome tics was indistinguishable from that of voluntary movements, low frequency activity in the thalamus could distinguish both, paving the way to closed-loop stimulation for Tourette syndrome.

Stanslaski S, Afshar P, Cong P, Giftakis J, Stypulkowski P, Carlson D, Linde D, Ullestad D, Avestruz AT, Denison T (2012) Design and validation of a fully implantable, chronic, closed-loop neuromodulation device with concurrent sensing and stimulation. IEEE Trans Neural Syst Rehabil Eng 20:410-421. 
Sun FT, Morrell MJ (2014) Closed-loop neurostimulation: the clinical experience. Neurotherapeutics 11:553-563.

Sun L, Hinrichs H (2016) Moving average template subtraction to remove stimulation artefacts in EEGs and LFPs recorded during deep brain stimulation. J Neurosci Methods 266:126-136.

Swann NC, de Hemptinne C, Miocinovic S, Qasim S, Wang SS, Ziman N, Ostrem JL, San Luciano M, Galifianakis NB, Starr PA (2016) Gamma oscillations in the hyperkinetic state detected with chronic human brain recordings in Parkinson's disease. J Neurosci 36:6445-6458.

(•) Swann NC, de Hemptinne C, Thompson MC, Miocinovic S, Miller AM, Gilron R, Ostrem JL, Chizeck HJ, Starr PA (2018) Adaptive deep brain stimulation for Parkinson's disease using motor cortex sensing. J Neural Eng 15:046006. Swann et al. showed for the first time how gamma activity in the motor cortex activity could be used as a marker of dyskinesia to reduce STN stimulation, saving battery while maintaining therapeutic efficacy.

Thies, M., Zrenner, C., Ziemann, U., \& Bergmann, T. O. (2018). Sensorimotor mu-power is positively related to corticospinal excitability. Brain Stimulation 11:1119-1122.

Thut G, Bergmann TO, Fröhlich F, Soekadar SR, Brittain JS, Valero-Cabré A, Sack AT, Miniussi C, Antal A, Siebner HR, Ziemann U, Herrmann CS (2017) Guiding transcranial brain stimulation by EEG/MEG to interact with ongoing brain activity and associated functions: a position paper. Clin Neurophysiol 128:843-857.

Thut G, Veniero D, Romei V, Miniussi C, Schyns P, Gross J (2011) Rhythmic TMS causes local entrainment of natural oscillatory signatures. Curr Biol 21:1176-1185.

(•) Tinkhauser G, Pogosyan A, Little S, Beudel M, Herz DM, Tan H, Brown P (2017) The modulatory effect of adaptive deep brain stimulation on beta bursts in Parkinson's disease. Brain 140:1053-1067.

The authors of this study were amongst the first to study the neurophysiological effects of closed-loop DBS on beta activity.

Tran, T. T., Hoffner, N. C., LaHue, S. C., Tseng, L., \& Voytek, B. (2016). Alpha phase dynamics predict age-related visual working memory decline. Neuroimage 143:196-203.

Trebaul L, Rudrauf D, Job AS, Mălîia MD, Popa I, Barborica A, Minotti L, Mîndruță I, Kahane P, David O (2016) Stimulation artifact correction method for estimation of early cortico-cortical evoked potentials. J Neurosci Methods 264:94-102.

Uhlhaas, P. J., \& Singer, W. (2006). Neural synchrony in brain disorders: relevance for cognitive dysfunctions and pathophysiology. Neuron 52:155-168.

Wang, D. D., de Hemptinne, C., Miocinovic, S., Qasim, S. E., Miller, A. M., Ostrem, J. L., ... \& Starr, P. A. (2016). Subthalamic local field potentials in Parkinson's disease and isolated dystonia: an evaluation of potential biomarkers. Neurobiology of Disease 89:213-222.

Wang S, Mamelak AN, Adolphs R, Rutishauser U (2018) Encoding of target detection during visual search by single neurons in the human brain. Curr Biol 28:1-12.

Witkowski M, Garcia-Cossio E, Chander BS, Braun C, Birbaumer N, Robinson SE, Soekadar SR (2016) Mapping entrained brain oscillations during transcranial alternating current stimulation (tACS). Neuroimage 140:89-98.

Zhou A, Johnson BC, Muller R (2018) Toward true closed-loop neuromodulation: artifact-free recording during stimulation. Curr Opin Neurobiol 50:119-127.

Zrenner, C., Desideri, D., Belardinelli, P., \& Ziemann, U. (2018). Real-time EEG-defined excitability states determine efficacy of TMS-induced plasticity in human motor cortex. Brain Stimulation 11:374-389.

(•) Zrenner, C., Belardinelli, P., Müller-Dahlhaus, F., \& Ziemann, U. (2016). Closed-loop neuroscience and non-invasive brain stimulation: a tale of two loops. Frontiers in Cellular Neuroscience 10:92.

In this opinion study, Zrenner et al. suggest that a closed-loop stimulation application may require the use of a second, taskrelated, closed-loop, similar to typical brain-machine interfaces. With this approach, they expect closed-loop approaches to lead to long-term efficacy and plastic changes due to metaplasticity. 